\title{
A cortical-hypothalamic circuit decodes social rank and promotes dominance behavior
}

\section{Nancy Padilla-Coreano}

Salk Institute

\section{Kanha Batra}

Salk Institute

\section{Makenzie Patarino}

Salk Institute

\section{Zexin Chen}

Shanghai Jiao Tong University Department of Computer Science

\section{Rachel Rock}

Salk Institute

Ruihan Zhang

MIT

\section{Sebastien Hausmann}

Salk Institute for Biological Studies

Javier Weddington

MIT

\section{Reesha Patel}

Salk Institute for Biological Studies

\section{Yu Zhang}

Salk Institute for Biological Studies

\section{Hao-Shu Fang}

Shanghai Jiao Tong University Department of Computer Science

\section{Laurel Keyes}

Salk Institute

\section{Avraham Libster}

Salk Institute for Biological Studies

\section{Gillian Matthews}

Salk Institute for Biological Studies

\section{James Curley}

University of Texas at Austin Department of Psychology

\section{Ila Fiete}

MIT https://orcid.org/0000-0003-4738-2539

Cewu Lu 
Shanghai Jiao Tong University Department of Computer Science

Kay Tye ( $\nabla$ tye@salk.edu )

Salk Institute https://orcid.org/0000-0002-2435-0182

\section{Biological Sciences - Article}

Keywords: cortical-hypothalamic circuit, social rank, dominance behavior, medial prefrontal cortex (mPFC)

Posted Date: November 3rd, 2020

DOl: https://doi.org/10.21203/rs.3.rs-94115/v1

License: (c) (1) This work is licensed under a Creative Commons Attribution 4.0 International License. Read Full License

Version of Record: A version of this preprint was published at Nature on March 16th, 2022. See the published version at https://doi.org/10.1038/s41586-022-04507-5. 


\section{Abstract}

How do we know our social rank? Most social species, from insects to humans, self-organize into social dominance hierarchies (1-4). The establishment of social ranks serves to decrease aggression, conserve energy, and maximize survival for the entire group (5-8). Despite dominance behaviors being critical for successful interactions and ultimately, survival, we have only begun to learn how the brain represents social rank (9-12) and guides behavior based on this representation. The medial prefrontal cortex (mPFC) has been implicated in the expression of social dominance in rodents $(10,11)$, and in social rank learning in humans $(13,14)$. Yet precisely how the mPFC encodes rank and which circuits mediate this computation is not known. We developed a trial-based social competition assay in which mice compete for rewards, as well as a computer vision tool to track multiple, unmarked animals. With the development of a deep learning computer vision tool (AlphaTracker) and wireless electrophysiology recording devices, we have established a novel platform to facilitate quantitative examination of how the brain gives rise to social behaviors. We describe nine behavioral states during social competition that were accurately decoded from mPFC ensemble activity using a hidden Markov model combined with generalized linear models (HMM-GLM). Population dynamics in the mPFC were predictive of social rank and competitive success. This population-level rank representation translated into differences in the individual cell responses to task-relevant events across ranks. Finally, we demonstrate that mPFC cells that project to the lateral hypothalamus contribute to the prediction of social rank and promote dominance behavior during the reward competition. Thus, we reveal a cortico-hypothalamic circuit by which mPFC exerts topdown modulation of social dominance.

\section{Main Text}

The medial prefrontal cortex (MPFC) is best known for its role in working memory, decision-making, reward learning and goal-oriented behavior ${ }^{15-19}$. Theories about mPFC function emphasize that it integrates sensory and limbic information to flexibly guide behavior based on task rules ${ }^{20,21}$. mPFC circuitry has also been broadly implicated in social cognition ${ }^{22-24}$, social behaviors ${ }^{25,26}$, social defeat $^{27,28}$, and social dominance ${ }^{10,11,14}$.

These conceptual frameworks, led us to hypothesize that mPFC neurons encode social rank and are part of top-down circuits to guide behavior based on social rank.

To determine if mPFC encodes social rank we needed to measure social dominance in an assay to facilitate statistical comparison. However, existing dominance assays, such as the tube test or the urine marking assay, lack the trial structure and have sensorimotor confounds ${ }^{11,12,29,30}$. To overcome these challenges, we considered that dominant animals often have priority access to resources. We designed an ethologically-relevant competition assay wherein mice that were linearly-ranked among their cage mates using the tube test ${ }^{11}$ (Fig. 1a) competed for a palatable liquid reward (Ensure) delivered two seconds after the onset of a tone. Throughout our analyses we considered social rank differences in each competing pair and thus refer to "dominant" and "subordinate" based on relative social ranks. After 
individually learning the tone-reward association (Fig. 1b), mice competed for the rewards with a cage mate (Fig. 1a). Dominant animals, as defined by the tube test, obtained more rewards, spent more time at the reward port, and were more successful at displacing the competitor from the port (Fig. 1c). Importantly, differences in winning were not driven by overall location in the arena or distance to port prior to tone onset (Extended Data Fig. 1). Therefore, this reward competition provides a trial-based behavioral paradigm for measuring dominance behavior facilitating statistical comparisons of neuronal firing rate.

To automatically track the behavior of multiple, unmarked mice we developed AlphaTracker, a deep learning tool based on AlphaPose ${ }^{31}$. AlphaTracker combines two neural networks, one to create a bounding box for each subject, another for pose estimation to detect multiple indistinguishable animals (Fig. 1d). AlphaTracker also applies an additional algorithm to track animal identity across frames by taking into consideration animal positions from the previous frame (Fig. 1d; see methods). The performance of AlphaTracker surpasses human accuracy and precision when using more than 100 frames for training (Fig.1e, Extended Data Fig. 2 and Supplementary Movie 1). In addition, the AlphaTracker package includes unsupervised clustering of the tracking output data to aid the identification of novel behavioral motifs (Fig. 1f-h, Extended Data Fig. 3a-f and Supplementary Movie 1) ${ }^{32}$. Furthermore, AlphaTracker performs well with low resolution videos and varying angles. Importantly, AlphaTracker can generalize to more animals even when training data sets included only two mice (Supplementary Movie 1). To correct potential identity tracking errors and review the behavioral clustering output, we created a graphical user interface (Extended Data Fig. $2 \mathrm{~b}$ and Supplementary Movie 1). The development and application of AlphaTracker represents a new platform for the emerging field of computational neuroethology of social behaviors ${ }^{33,34}$.

\section{mPFC neural activity can be decoded to predict precise behavioral state}

We sought to investigate whether the MPFC encodes the behavioral states observed during social competition. The use of wireless head-mounted devices, as opposed to tethering, minimizes the impact on social behavior ${ }^{35}$. We recorded cellular resolution activity from the mPFC using a wireless headmounted electrophysiological recording device (Fig. 2a and Extended Data Fig. 4). When recording during the reward competition task, we did not detect a statistically significant difference in the number of rewards earned by dominant and subordinate mice, allowing us to make comparisons about dominance behavior and competitive success without being confounded by the volume of reward consumption. Importantly, dominant mice had higher pushing success while subordinates collected rewards with a longer latency (Extended Data Fig. 5a-d). AlphaTracker enabled quantification of a variety of behavioral states that mice expressed during the reward competition (Extended Data Fig. 5e-f). We trained a multinomial support vector machine (SVM) classifier to decode these behavioral states using mPFC multi-unit activity (Fig. 2d). However, we considered that the static nature of an SVM may not fully capture the relationship between mPFC neural activity and behavior.

We posited that mPFC neural activity could be dynamic and may be influenced by internal hidden states. Therefore, we turned to a recently-developed unsupervised method to identify hidden states by combining 
a hidden Markov model (HMM) with generalized linear models (GLMs) ${ }^{36,37}$ and adapted it to use mPFC neural activity to predict each of nine behavioral states. We trained a set of multinomial GLMs to predict the transition probabilities between hidden states. In addition, each hidden state is paired with another multinomial GLM that describes the relationship between neural activity and the behavior of that particular hidden state (Fig. 2a-b).

To create a model of the temporal relationship between neural activity and behavior with performance superior to that of static models, each component of our dynamic model followed the first-order Markovian property, to help preserve information about past events when predicting the future (Fig. 2d). An HMM-GLM model with 6 hidden states decoded behavioral state from neural activity with the superior performance to static models (Fig. 2c-e, Extended Data Fig. 6a-b and Supplementary Movie 2). However, the proportion of time spent in each hidden state did not differ by competitive success or by social rank, and the model performed equally well across ranks (Extended Data Fig. 6c-e), suggesting that mPFC encoding of social competition behavioral states is common across ranks.

\section{mPFC neural activity stably represents rank and predicts future competitive success}

We then wondered whether MPFC neural activity could be used to decode social rank, and if the neural representation of social rank is triggered by discrete events (such as cued competition trials) or stable throughout the task. To visualize population activity, we plotted the population activity vector for taskrelevant events (Extended Data Fig. 7a; Supplementary Movie 3) in a lower dimensional state-space using principal component analysis $(\mathrm{PCA})^{38-41}$. To compare the population dynamics across ranks, we found a common two-dimensional PCA for all animals pooled, and subsequently plotted the evolving lowerdimensional population firing rates (i.e. neural trajectories) for dominants and subordinates in this subspace. Neural trajectories during winning and losing occupied segregated PCA subspaces - even before the cue onset (Fig. 3a; Extended Data Fig. 7b). To reveal differences in population dynamics across rank, we quantified the length of the neural trajectories ${ }^{42}$ for win and lose trials and found that subordinates had longer neural trajectories, suggesting either higher or faster firing rate changes in the mPFC population activity (Fig. 3b). Interestingly, when we analyzed the win and lose subspaces separately by performing the PCA for each event, we observed that the win and lose neural trajectories were less aligned in dominant mice, suggesting that the ensemble activity differences between winning and losing are larger in dominants than in subordinates (Extended Data Fig. 7c). Similar to win and lose trials, the MPFC population dynamics for reward port entries of self and other during the tone (Fig. 3c-d) and during the inter-trial intervals (Extended Data Fig. 7d-f) were non-overlapping in the state-space, and the subordinate neural trajectories were longer (Fig. 3d, Extended Data Fig. 7f). Overall, there was segregation of the ensemble representation of actions of self $\mathrm{vs}$ other and rank in mPFC population dynamics across the subspaces (Fig. 3e).

To rule out the possibility that any rank-related differences in win and lose neural trajectories could be explained by differences in spatial location or distance to the reward port (Fig. 3f-g), we calculated the trajectory lengths for trials when mice were close to, or far from, the reward port, separately. In both 
cases, we found that subordinate trajectories were longer (Extended Data Fig. 7g-h), consistent with more dynamic mPFC activity regardless of distance to port. In addition, we identified single units that were correlated with distance to the reward port (Fig. 3h) and when we excluded them from the port entry neural trajectories, subordinates still had longer population trajectories (Extended Data Fig. 7i-j). Splitting the data into two subsets of random animals did not yield detectable differences in the neural trajectories across the two groups (Extended Data Fig. 7k-n), suggesting that the population dynamics observed across ranks are not due to differences in social identity.

To directly test the hypothesis that mPFC encodes rank and competitive success at the population level, we trained a support vector machine (SVM) classifier to decode competitive success as well as relative social rank from single-trial data (Fig. 3i). Consistent with the linear separation seen in the first 2 PCs (Fig. 3e), a linear SVM was able to decode rank and competitive success, however, an SVM with a kernel (which increases dimensionality) performed significantly better (Fig. 3j, Extended Data Fig. 8a). Altogether, these data demonstrate that the MPFC has a stable representation of social rank and competitive success despite having multiple, rank-independent hidden states for encoding behavioral states during social competition.

Interestingly, social rank and competitive success could be decoded accurately prior to cue onset (Extended Data Fig. 8b; Fig. 3) consistent with the notion that state differences in mPFC correlate with future winning ${ }^{10}$. Social rank was more accurately decoded than competition outcome from mPFC neural activity (Fig. 3k), which may reflect the relative stability of rank versus competitive success. While the idea of a "winning effect" or a "losing streak" is not novel, ${ }^{10,43-45}$ we were surprised that the decoding accuracy was stable across the trial, with the MPFC representing future competitive success with the same accuracy as during the actual competition (Fig. 3j). Remarkably, we could accurately predict whether the next trial would be a win or a loss more than 30 seconds before the competition trial began, providing empirical evidence at the cellular level supporting the psychological concept of "a winning mindset." While mPFC activity of dominant mice predicted competitive success with the same accuracy before and during the trial (Extended Data Fig. 8c), subordinate mice showed a significant increase in decoder performance of competition outcome once the trial was initiated (Extended Data Fig. 8d).

\section{mPFC single units show rank-dependent responses to task-relevant events during social competition}

To determine if the MPFC representation of rank was also present at the single-cell level, we analyzed the firing rate of mPFC single units during task-relevant events of the reward competition. mPFC single units showed responses to the tone for win or lose trials and to port entries performed by self or the other (i.e. competitor) that differed by social rank (Fig. 4a). To identify potential activity patterns linked to dominance, we performed unsupervised clustering using the single unit firing rate during the task-relevant events for all animals pooled (Fig. 4b; Extended Data Fig. 9a-b) and separated the data by relative social rank post-clustering. Next, we quantified the distribution of dominant vs subordinate mPFC cells across functional clusters and identified several clusters that were enriched in a rank-dependent manner (Fig. 4ce). Subordinate mPFC cells showed strong excitation to win trials and port entries of the other (clusters 3 
\& 5), whereas dominant mPFC cells were more likely to respond to win trials and port entries of the self (clusters $4 \& 8$ ). As suggested by these differences in the functional clusters, the dominant and subordinate mPFC cells responded differently to competition outcome and reward port entries. Dominant mice had more cells that were responsive to self port entries while subordinate mice had larger responses to win trials and port entries of the other (Fig. 4f-i; Extended Data Fig. 9c-f). Surprisingly, subordinates had phasic responses of greater amplitude in response to events, which is consistent with subordinates having longer neural trajectories. Given the functional diversity of neural responses from individual mPFC neurons, we next wanted to investigate how information was routed out of the mPFC during social competition.

\section{mPFC-LH neurons encode social rank and modulate dominance behavior}

Next, we sought to investigate which subcortical-projecting mPFC subpopulations contributed to the encoding of social rank during social competition. Several MPFC subcortical pathways have been implicated in social behaviors ${ }^{46}$. Among these, we investigated the downstream projection to the lateral hypothalamus $(\mathrm{LH})$, as it is the most prominent prefrontal top-down circuit to the hypothalamus and modulates social exploration ${ }^{47}$, defensive behaviors ${ }^{48,49}$, and reward and aversion ${ }^{47,50-52}$. We also investigated the MPFC projection to the basolateral amygdala (BLA), because recent evidence suggests that BLA firing rates correlate with the social rank of conspecific faces in non-human primates ${ }^{53}$ and the BLA is an important point of convergence for socially-derived information ${ }^{54}$ to be associated with emotional valence ${ }^{26,54-56}$.

To selectively activate mPFC cells that project monosynaptically to the LH or BLA, we used an intersectional viral strategy to express ChrimsonR ${ }^{57}$ in each projection (Fig. 5a-b, Extended Data Fig. 10ab). Ex vivo, mPFC-LH and mPFC-BLA cells fired action potentials with photo-response latencies of less than $8 \mathrm{~ms}$ to red light, while non-expressing neighbors only had subthreshold activations that were slower, allowing us to use this photo-response latency threshold for photoidentification ${ }^{58}$. Therefore in vivo phototagging of these subpopulations is permissible with an appropriate photo-response latency threshold (Fig 5a-b). We then recorded mPFC single units wirelessly during the reward competition and delivered red light pulses at the end of the competition to photo-identify mPFC-LH or MPFC-BLA neurons (Fig. 5c-d). We found that more mPFC-LH neurons were responsive to port entries done by the self compared to non-phototagged units, while there was no difference between MPFC-BLA neurons and nonphototagged units (Fig. 5e).

To determine whether these mPFC subpopulations contributed to the encoding of social rank or competitive success, we used an SVM classifier and removed a subpopulation prior to training the classifier or removed a matched number of non-phototagged cells. Removing mPFC-LH neurons, but not mPFC-BLA neurons, significantly decreased the performance of decoding social rank (Fig. 5f). Conversely, decoding of competitive success was unaffected by the removal of MPFC-LH neurons from the training data set, but was improved when removing the mPFC-BLA neurons (Fig. 5g). Importantly, mPFC-LH neurons did not contribute to the encoding of competitive success, suggesting that reward prediction and 
social rank could be encoded by different subpopulations in the mPFC. Altogether, these data suggest that mPFC-LH, but not mPFC-BLA, neurons contribute to the encoding of social rank in the mPFC.

To directly test the hypothesis that MPFC-LH neurons modulate social dominance-related behavior, we expressed either ChR2 or eYFP in MPFC-LH neurons and implanted an optic fiber in the mPFC (Fig. 5h; Extended Data Fig. 10b-c). After mice learned the tone-reward association, we performed the reward competition assay two days in a row: one day with no light and the other day with light delivery to the relative subordinate mouse in the pair during 5 minute on/off epochs consisting of 5 ms pulses at $100 \mathrm{~Hz}$ every $200 \mathrm{~ms}$ (Fig. 5i). ChR2-expressing mice won more rewards during the entire competition, had higher reward port occupation, and spent less time being displaced from the reward port when they received optical stimulation (Fig. 5j-m). Importantly, stimulating mPFC-LH neurons did not affect behavior while performing the reward competition assay alone (Extended Data Fig. 10d). In addition, mPFC-LH stimulation did not affect food consumption, sociability, anxiety-like behavior, or conditioned place preference (Extended Data Fig. 10e-h). Altogether these data suggest that mPFC-LH stimulation increases social dominance behavior rather than affecting hunger or other motivated behaviors. An alternative interpretation of these results is that MPFC-LH neurons are increasing effort behavior, resulting in more won trials during the competition. Indeed, there is literature suggesting that the MPFC is necessary for effort-based decision making ${ }^{59}$. To test this hypothesis we used an effort-choice based T-maze paradigm ${ }^{59-61}$ in which mice choose between a low-effort low-reward arm or a high-effort high-reward arm in which they have to climb a wall to obtain the bigger reward (Extended Data Fig. 10i). Stimulation of mPFC-LH neurons did not increase effort as measured by the number of high effort choices (Extended Data Fig. 10j), indicating that a general increase in effort does not explain the increase in winning observed with stimulation of mPFC-LH neurons during the social competition.

Taken together, these data demonstrate that the MPFC neural activity predicts future competitive success, can be decoded to predict social rank and uses top-down circuits to flexibly guide social behaviors. Interestingly, although mPFC neurons encode social rank, the way they predicted behavioral states was independent of rank, suggesting that a common coding rule guides social behaviors during competition. Importantly, the way that mPFC ensembles encode behavior is dynamic, which suggests a model in which internal states influence how mPFC modulates behavior, consistent with a role in flexibly guiding behavior. Our data demonstrate that cortico-hypothalamic circuits carry social rank information which could potentially modulate the many different neuropeptide and hormone expressing subpopulations in the hypothalamus to achieve behavioral modulation based on social rank.

Considering the critical role for rank identification in interpreting the quality/quantity of social contact from a given social gesture, these data help to complete and synthesize previous findings. Here, we find that the $\mathrm{MPFC}$ plays a critical role in representing dominance, and confidence in future competitive success. We previously reported that anterior cingulate cortex (ACC) circuits play a critical role in observational learning $23,54,62,63$. These related circuits show complementary, but distinct functions, as the ACC-BLA circuit is critical for observational learning ${ }^{54}$, and the MPFC-BLA projection is involved in 
anxiety ${ }^{64}$, the mPFC-BLA projection is not critically involved in the representation of social rank (Fig. 5f) nor competitive success (Fig. $5 \mathrm{~g}$ ). We speculate both the ACC and mPFC contribute heavily to the detection of social contact and the evaluation of social rank through reciprocal loops between ACC and mPFC. Based on the known connectivity of the MPFC to dorsal raphe nucleus (DRN) dopamine neurons through both direct, reciprocal connections, and through indirect connection via the lateral hypothalamus ${ }^{65}$ (mPFC-LH-DRN), we speculate that mPFC circuits contribute heavily to the "social rank" node within a social homeostatic system ${ }^{66,67}$. Further, with the LH upstream of dopamine neurons that represent the cellular substrate for social reward (VTA) ${ }^{68}$ and a loneliness-like state (DRN) ${ }^{66}$, we speculate that the LH may represent a control center that can access these different downstream effector systems when either a surplus or deficit in social contact is detected.

This study not only unveils a number of technological advances that together provide a platform for the investigation of social hierarchies, but also connects a number of disparate pieces of information that support the notion that there is a neural circuit for social homeostasis.

\section{Methods}

Due to technical difficulties, the Methods section for this manuscript can only be viewed as a donwload in the Supplemental Files section.

\section{Declarations}

\section{Author Contributions:}

K.M.T., N.P.C. and K.B. conceptualized the project. N.P.C. and K.M.T. designed the experiments and supervised all experiments and data analyses. N.P.C. drafted the manuscript. N.P.C., K.M.T., M.P., R.R.R. and K.B. contributed to writing the manuscript and creating the figures. N.P.C., M.P., J.W., R.R.R., S.H., R.P., collected and analyzed data. K.B. created the HMM-GLM model and assisted with additional machine learning analyses in the manuscript. Z.C. and H.F. created AlphaTracker and assisted in the implementation of tracking and behavioral clustering under the supervision of C.L. R.Z. wrote code and implemented AlphaTracker behavioral clustering. Y.E.Z. and L.R.K. contributed to data analyses. N.P.C., K.B., G.M., J.P.C., I.R.F., C.L., A.L., R.Z., and K.M.T. made significant intellectual contributions.

\section{Acknowledgements:}

We thank Romy Wichmann, Craig Wiles, Radames Revilla Orellano, Sunny Luo and Crystal Chang for technical support. Adam Calhoun and Mala Murthy for useful feedback on our HMM-GLM model. We thank Ziv Williams for comments on our manuscript. K.M.T. is the Wylie Vale chair at Salk Institute for Biological Studies, a New York Stem Cell Foundation - Robertson Investigator and McKnight Scholar and this work was supported by funding from the JPB Foundation, Dolby Family Fund, R01-MH115920 
(NIMH), and Pioneer Award DP1-AT009925 (NCCIH). N.P.C. was supported by the Simons Center for the Social Brain, Ford Foundation and K99 MH124435-01.

\section{References}

1. Oliveira, R. F. \& Almada, V. C. Dominance hierarchies and social structure in captive groups of the Mozambique tilapia Oreochromis mossambicus (Teleostei Cichlidae). Ethol. Ecol. Evol. 8, 39-55 (1996).

2. Fewell, J. H. Social insect networks. Science 301, 1867-1870 (2003).

3. Chiao, J. Y. Neural basis of social status hierarchy across species. Curr. Opin. Neurobiol. 20, 803-809 (2010).

4. Wang, F., Kessels, H. W. \& Hu, H. The mouse that roared: neural mechanisms of social hierarchy. Trends Neurosci. 37, 674-682 (2014).

5. Barnard, C. J. \& Burk, T. Dominance hierarchies and the evolution of "individual recognition". J. Theor. Biol. 81, 65-73 (1979).

6. Bernstein, I. S. Dominance: The baby and the bathwater. Behav. Brain Sci. 4, 419-457 (1981).

7. Dewsbury, D. A. Dominance Rank, Copulatory Behavior, and Differential Reproduction. Q. Rev. Biol. 57, 135-159 (1982).

8. Karamihalev, S., Brivio, E., Flachskamm, C., Stoffel, R., Schmidt, M. V. \& Chen, A. Social dominance mediates behavioral adaptation to chronic stress in a sex-specific manner. eL ife 9 , e58723 (2020).

9. Hou, X. H., Hyun, M., Taranda, J., Huang, K. W., Todd, E., Feng, D., Atwater, E., Croney, D., Zeidel, M. L., Osten, P. \& Sabatini, B. L. Central Control Circuit for Context-Dependent Micturition. Cell 167, 7386.e12 (2016).

10. Zhou, T., Zhu, H., Fan, Z., Wang, F., Chen, Y., Liang, H., Yang, Z., Zhang, L., Lin, L., Zhan, Y., Wang, Z. \& $\mathrm{Hu}, \mathrm{H}$. History of winning remodels thalamo-PFC circuit to reinforce social dominance. Science 357 , 162-168 (2017).

11. Wang, F., Zhu, J., Zhu, H., Zhang, Q., Lin, Z. \& Hu, H. Bidirectional control of social hierarchy by synaptic efficacy in medial prefrontal cortex. Science 334, 693-697 (2011).

12. So, N., Franks, B., Lim, S. \& Curley, J. P. A Social Network Approach Reveals Associations between Mouse Social Dominance and Brain Gene Expression. PLOS ONE 10, e0134509 (2015).

13. Zink, C. F., Tong, Y., Chen, Q., Bassett, D. S., Stein, J. L. \& Meyer-Lindenberg, A. Know your place: neural processing of social hierarchy in humans. Neuron 58, 273-283 (2008).

14. Ligneul, R., Obeso, I., Ruff, C. C. \& Dreher, J.-C. Dynamical Representation of Dominance Relationships in the Human Rostromedial Prefrontal Cortex. Curr. Biol. 26, 3107-3115 (2016).

15. Sawaguchi, T. \& Goldman-Rakic, P. S. D1 dopamine receptors in prefrontal cortex: involvement in working memory. Science 251, 947-951 (1991).

16. Miller, E. K., Erickson, C. A. \& Desimone, R. Neural mechanisms of visual working memory in prefrontal cortex of the macaque. J. Neurosci. 16, 5154-5167 (1996). 
17. Wallis, J. D., Anderson, K. C. \& Miller, E. K. Single neurons in prefrontal cortex encode abstract rules. Nature 411, 953-956 (2001).

18. Hornak, J., O'doherty, J., Bramham, J., Rolls, E. T., Morris, R. G., Bullock, P. R. \& Polkey, C. E. Rewardrelated reversal learning after surgical excisions in orbito-frontal or dorsolateral prefrontal cortex in humans. J. Cogn. Neurosci. 16, 463-478 (2004).

19. Ridderinkhof, K. R., Van Den Wildenberg, W. P., Segalowitz, S. J. \& Carter, C. S. Neurocognitive mechanisms of cognitive control: the role of prefrontal cortex in action selection, response inhibition, performance monitoring, and reward-based learning. Brain Cogn. 56, 129-140 (2004).

20. Miller, E. K. The prefrontal cortex: complex neural properties for complex behavior. Neuron 22, 15-17 (1999).

21. Miller, E. K. \& Cohen, J. D. An integrative theory of prefrontal cortex function. Annu. Rev. Neurosci. 24, 167-202 (2001).

22. Levy, D. R., Tamir, T., Kaufman, M., Parabucki, A., Weissbrod, A., Schneidman, E. \& Yizhar, O. Dynamics of social representation in the mouse prefrontal cortex. Nat. Neurosci. 22, 2013-2022 (2019).

23. Apps, M. A. J., Rushworth, M. F. S. \& Chang, S. W. C. The Anterior Cingulate Gyrus and Social Cognition: Tracking the Motivation of Others. Neuron 90, 692-707 (2016).

24. Murugan, M., Jang, H. J., Park, M., Miller, E. M., Cox, J., Taliaferro, J. P., Parker, N. F., Bhave, V., Hur, H., Liang, Y., Nectow, A. R., Pillow, J. W. \& Witten, I. B. Combined Social and Spatial Coding in a Descending Projection from the Prefrontal Cortex. Cell 171, 1663-1677.e16 (2017).

25. Yizhar, O., Fenno, L. E., Prigge, M., Schneider, F., Davidson, T. J., O’Shea, D. J., Sohal, V. S., Goshen, I., Finkelstein, J., Paz, J. T., Stehfest, K., Fudim, R., Ramakrishnan, C., Huguenard, J. R., Hegemann, P. \& Deisseroth, $\mathrm{K}$. Neocortical excitation/inhibition balance in information processing and social dysfunction. Nature (2011). doi:10.1038/nature10360

26. Felix-Ortiz, A. C., Burgos-Robles, A., Bhagat, N. D., Leppla, C. A. \& Tye, K. M. Bidirectional modulation of anxiety-related and social behaviors by amygdala projections to the medial prefrontal cortex. Neuroscience 321, 197-209 (2016).

27. Kumar, S., Hultman, R., Hughes, D., Michel, N., Katz, B. M. \& Dzirasa, K. Prefrontal cortex reactivity underlies trait vulnerability to chronic social defeat stress. Nat. Commun. 5, 4537 (2014).

28. Mague, S. D., Talbot, A., Blount, C., Duffney, L. J., Walder-Christensen, K. K., Adamson, E., Bey, A. L., Ndubuizu, N., Thomas, G., Hughes, D. N., Sinha, S., Fink, A. M., Gallagher, N. M., Fisher, R. L., Jiang, Y., Carlson, D. E. \& Dzirasa, K. Brain-wide electrical dynamics encode an appetitive socioemotional state. bioRxiv 2020.07.01.181347 (2020). doi:10.1101/2020.07.01.181347

29. Lindzey, G., Winston, H. \& Manosevitz, M. Social Dominance in Inbred Mouse Strains. Nature 191, 474 (1961).

30. Desjardins, C., Maruniak, J. A. \& Bronson, F. H. Social Rank in House Mice: Differentiation Revealed by Ultraviolet Visualization of Urinary Marking Patterns. Science 182, 939-941 (1973).

31. Fang, H.-S., Xie, S., Tai, Y.-W. \& Lu, C. RMPE: Regional Multi-person Pose Estimation. in 2017 IEEE Int. Conf. Comput. Vis. ICCV 2353-2362 (IEEE, 2017). doi:10.1109/ICCV.2017.256 
32. Wiltschko, A. B., Johnson, M. J., Iurilli, G., Peterson, R. E., Katon, J. M., Pashkovski, S. L., Abraira, V. E., Adams, R. P. \& Datta, S. R. Mapping Sub-Second Structure in Mouse Behavior. Neuron 88, 11211135 (2015).

33. Pereira, T. D., Tabris, N., Li, J., Ravindranath, S., Papadoyannis, E. S., Wang, Z. Y., Turner, D. M., McKenzie-Smith, G., Kocher, S. D., Falkner, A. L., Shaevitz, J. W. \& Murthy, M. SLEAP: Multi-animal pose tracking. bioRxiv 2020.08.31.276246 (2020). doi:10.1101/2020.08.31.276246

34. Nilsson, S. R., Goodwin, N. L., Choong, J. J., Hwang, S., Wright, H. R., Norville, Z. C., Tong, X., Lin, D., Bentzley, B. S., Eshel, N., McLaughlin, R. J. \& Golden, S. A. Simple Behavioral Analysis (SimBA) - an open source toolkit for computer classification of complex social behaviors in experimental animals. bioRxiv 2020.04.19.049452 (2020). doi:10.1101/2020.04.19.049452

35. Anpilov, S., Shemesh, Y., Eren, N., Harony-Nicolas, H., Benjamin, A., Dine, J., Oliveira, V. E. M., Forkosh, O., Karamihalev, S., Hüttl, R.-E., Feldman, N., Berger, R., Dagan, A., Chen, G., Neumann, I. D., Wagner, S., Yizhar, O. \& Chen, A. Wireless Optogenetic Stimulation of Oxytocin Neurons in a Semi-natural Setup Dynamically Elevates Both Pro-social and Agonistic Behaviors. Neuron 107, 644-655.e7 (2020).

36. Escola, S., Fontanini, A., Katz, D. \& Paninski, L. Hidden Markov models for the stimulus-response relationships of multistate neural systems. Neural Comput. 23, 1071-1132 (2011).

37. Calhoun, A. J., Pillow, J. W. \& Murthy, M. Unsupervised identification of the internal states that shape natural behavior. Nat. Neurosci. 22, 2040-2049 (2019).

38. Cunningham, J. P. \& Yu, B. M. Dimensionality reduction for large-scale neural recordings. Nat. Neurosci. 17, 1500-1509 (2014).

39. Gao, P. \& Ganguli, S. On simplicity and complexity in the brave new world of large-scale neuroscience. Curr. Opin. Neurobiol. 32, 148-155 (2015).

40. Linsenbardt, D. N., Timme, N. M. \& Lapish, C. C. Encoding of the Intent to Drink Alcohol by the Prefrontal Cortex Is Blunted in Rats with a Family History of Excessive Drinking. eNeuro 6, (2019).

41. Williamson, R. C., Doiron, B., Smith, M. A. \& Yu, B. M. Bridging large-scale neuronal recordings and large-scale network models using dimensionality reduction. Curr. Opin. Neurobiol. 55, 40-47 (2019).

42. Shifrin, T. Differential geometry: a first course in curves and surfaces. Univ. Ga. (2015).

43. Mesterton-Gibbons, M. On the evolution of pure winner and loser effects: A game-theoretic model. Bull. Math. Biol. 61, 1151-1186 (1999).

44. Bonabeau, E., Theraulaz, G. \& Deneubourg, J.-L. Dominance orders in animal societies: The selforganization hypothesis revisited. Bull. Math. Biol. 61, 727-757 (1999).

45. Kura, K., Broom, M. \& Kandler, A. A Game-Theoretical Winner and Loser Model of Dominance Hierarchy Formation. Bull. Math. Biol. 78, 1259-1290 (2016).

46. Ko, J. Neuroanatomical Substrates of Rodent Social Behavior: The Medial Prefrontal Cortex and Its Projection Patterns. Front. Neural Circuits 11, (2017).

47. Nieh, E. H., Vander Weele, C. M., Matthews, G. A., Presbrey, K. N., Wichmann, R., Leppla, C. A., Izadmehr, E. M. \& Tye, K. M. Inhibitory Input from the Lateral Hypothalamus to the Ventral Tegmental 
Area Disinhibits Dopamine Neurons and Promotes Behavioral Activation. Neuron 90, 1286-1298 (2016).

48. Rangel, M. J., Baldo, M. V. C., Canteras, N. S. \& Hahn, J. D. Evidence of a Role for the Lateral Hypothalamic Area Juxtadorsomedial Region (LHAjd) in Defensive Behaviors Associated with Social Defeat. Front. Syst. Neurosci. 10, 92 (2016).

49. Li, Y., Zeng, J., Zhang, J., Yue, C., Zhong, W., Liu, Z., Feng, Q. \& Luo, M. Hypothalamic circuits for predation and evasion. Neuron 97, 911-924 (2018).

50. Aston-Jones, G., Smith, R. J., Moorman, D. E. \& Richardson, K. A. Role of lateral hypothalamic orexin neurons in reward processing and addiction. Neuropharmacology 56 Suppl 1, 112-121 (2009).

51. Jennings, J. H., Ung, R. L., Resendez, S. L., Stamatakis, A. M., Taylor, J. G., Huang, J., Veleta, K., Kantak, P. A., Aita, M., Shilling-Scrivo, K., Ramakrishnan, C., Deisseroth, K., Otte, S. \& Stuber, G. D. Visualizing hypothalamic network dynamics for appetitive and consummatory behaviors. Cel/ 160, 516-527 (2015).

52. Nieh, E. H., Matthews, G. A., Allsop, S. A., Presbrey, K. N., Leppla, C. A., Wichmann, R., Neve, R., Wildes, C. P. \& Tye, K. M. Decoding Neural Circuits that Control Compulsive Sucrose Seeking. Cel/ 160, 528541 (2015).

53. Munuera, J., Rigotti, M. \& Salzman, C. D. Shared neural coding for social hierarchy and reward value in primate amygdala. Nat. Neurosci. 21,415-423 (2018).

54. Allsop, S. A., Wichmann, R., Mills, F., Burgos-Robles, A., Chang, C.-J., Felix-Ortiz, A. C., Vienne, A., Beyeler, A., Izadmehr, E. M., Glober, G., Cum, M. I., Stergiadou, J., Anandalingam, K. K., Farris, K., Namburi, P., Leppla, C. A., Weddington, J. C., Nieh, E. H., Smith, A. C., Ba, D., Brown, E. N. \& Tye, K. M. Corticoamygdala Transfer of Socially Derived Information Gates Observational Learning. Cell 173, 1329-1342.e18 (2018).

55. Burgos-Robles, A., Kimchi, E. Y., Izadmehr, E. M., Porzenheim, M. J., Ramos-Guasp, W. A., Nieh, E. H., Felix-Ortiz, A. C., Namburi, P., Leppla, C. A., Presbrey, K. N., Anandalingam, K. K., Pagan-Rivera, P. A., Anahtar, M., Beyeler, A. \& Tye, K. M. Amygdala inputs to prefrontal cortex guide behavior amid conflicting cues of reward and punishment. Nat. Neurosci. 20, 824-835 (2017).

56. Janak, P. H. \& Tye, K. M. From circuits to behaviour in the amygdala. Nature 517, 284-292 (2015).

57. Klapoetke, N. C., Murata, Y., Kim, S. S., Pulver, S. R., Birdsey-Benson, A., Cho, Y. K., Morimoto, T. K., Chuong, A. S., Carpenter, E. J., Tian, Z., Wang, J., Xie, Y., Yan, Z., Zhang, Y., Chow, B. Y., Surek, B., Melkonian, M., Jayaraman, V., Constantine-Paton, M., Wong, G. K.-S. \& Boyden, E. S. Independent optical excitation of distinct neural populations. Nat. Methods 11, 338-346 (2014).

58. Beyeler, A., Namburi, P., Glober, G. F., Simonnet, C., Calhoon, G. G., Conyers, G. F., Luck, R., Wildes, C. P. \& Tye, K. M. Divergent Routing of Positive and Negative Information from the Amygdala during Memory Retrieval. Neuron 90, 348-361 (2016).

59. Walton, M. E., Bannerman, D. M. \& Rushworth, M. F. The role of rat medial frontal cortex in effortbased decision making. J. Neurosci. 22, 10996-11003 (2002). 
60. Floresco, S. B. \& Ghods-Sharifi, S. Amygdala-Prefrontal Cortical Circuitry Regulates Effort-Based Decision Making. Cereb. Cortex 17, 251-260 (2007).

61. Rudebeck, P. H., Walton, M. E., Smyth, A. N., Bannerman, D. M. \& Rushworth, M. F. S. Separate neural pathways process different decision costs. Nat. Neurosci. 9, 1161-1168 (2006).

62. Basile, B. M., Schafroth, J. L., Karaskiewicz, C. L., Chang, S. W. C. \& Murray, E. A. The anterior cingulate cortex is necessary for forming prosocial preferences from vicarious reinforcement in monkeys. PLOS Biol. 18, e3000677 (2020).

63. Jeon, D., Kim, S., Chetana, M., Jo, D., Ruley, H. E., Lin, S.-Y., Rabah, D., Kinet, J.-P. \& Shin, H.-S. Observational fear learning involves affective pain system and Cav1.2 Ca2+ channels in ACC. Nat. Neurosci. 13, 482-488 (2010).

64. Likhtik, E., Stujenske, J. M., Topiwala, M. A., Harris, A. Z. \& Gordon, J. A. Prefrontal entrainment of amygdala activity signals safety in learned fear and innate anxiety. Nat. Neurosci. 17, 106-113 (2014).

65. Cardozo Pinto, D. F., Yang, H., Pollak Dorocic, I., de Jong, J. W., Han, V. J., Peck, J. R., Zhu, Y., Liu, C., Beier, K. T., Smidt, M. P. \& Lammel, S. Characterization of transgenic mouse models targeting neuromodulatory systems reveals organizational principles of the dorsal raphe. Nat. Commun. 10, 4633 (2019).

66. Matthews, G. A., Nieh, E. H., Vander Weele, C. M., Halbert, S. A., Pradhan, R. V., Yosafat, A. S., Glober, G. F., Izadmehr, E. M., Thomas, R. E., Lacy, G. D., Wildes, C. P., Ungless, M. A. \& Tye, K. M. Dorsal Raphe Dopamine Neurons Represent the Experience of Social Isolation. Cell 164, 617-631 (2016).

67. Matthews, G. A. \& Tye, K. M. Neural mechanisms of social homeostasis. Ann. N. Y. Acad. Sci. 1457, 5-25 (2019).

68. Nieh, E. H., Vander Weele, C. M., Matthews, G. A., Presbrey, K. N., Wichmann, R., Leppla, C. A., Izadmehr, E. M. \& Tye, K. M. Inhibitory Input from the Lateral Hypothalamus to the Ventral Tegmental Area Disinhibits Dopamine Neurons and Promotes Behavioral Activation. Neuron 90, 1286-1298 (2016).

\section{Figures}



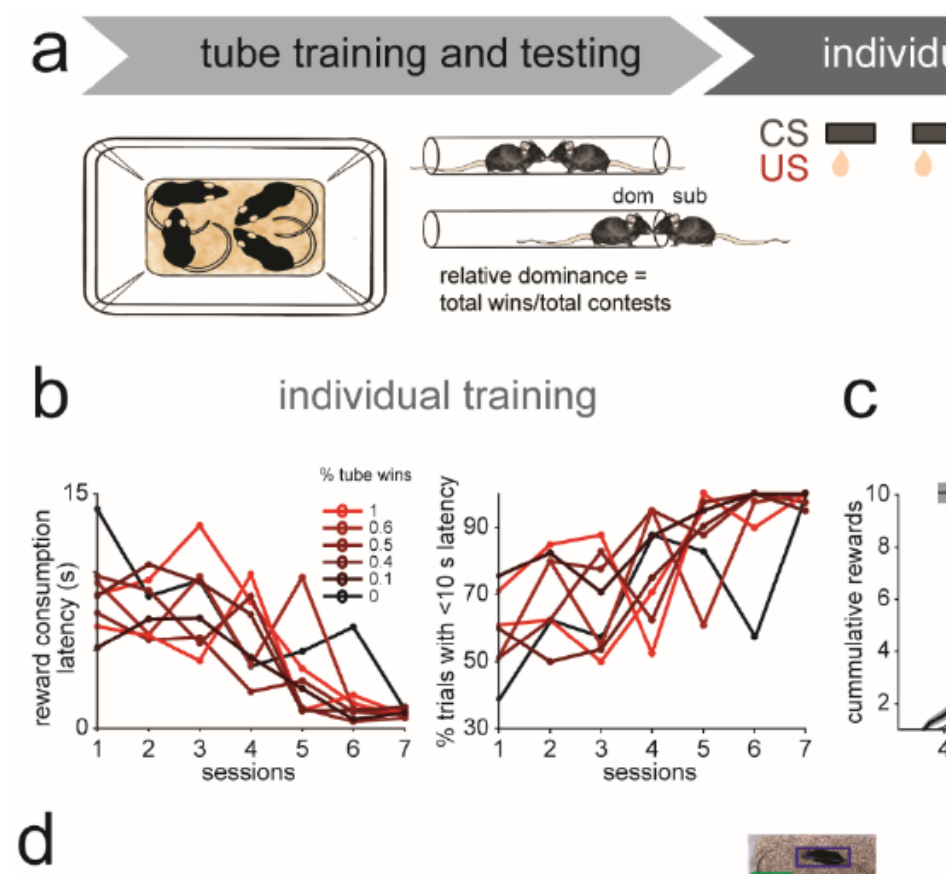

C
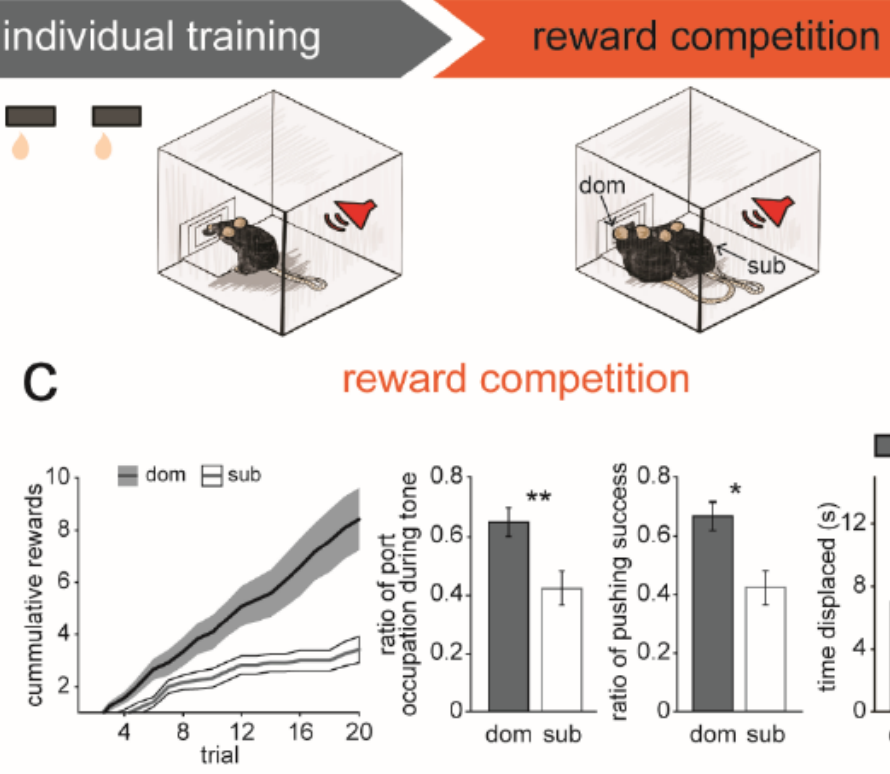

reward competition
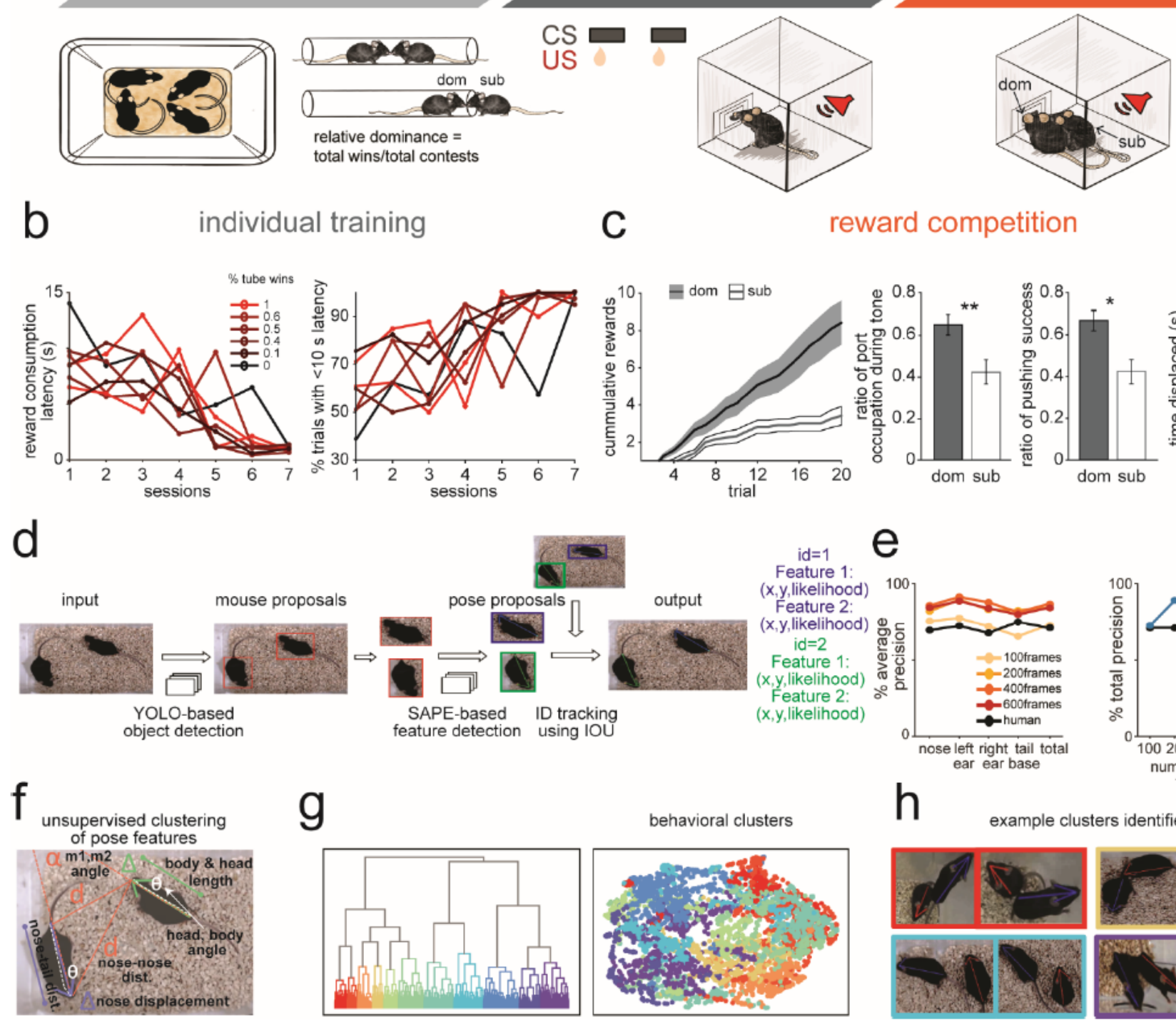

behavioral clusters
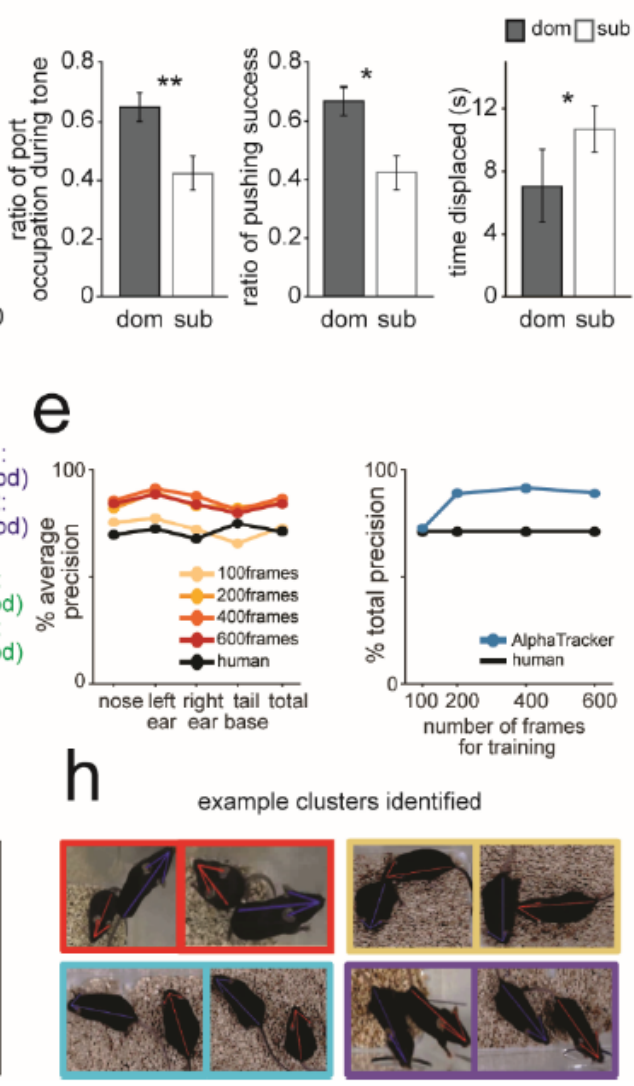

\section{Figure 1}

Novel social dominance assay and deep learning tool for tracking multiple animals a. Schematic of reward competition assay behavioral paradigm. First, social rank was assayed using the tube test. Once ranks were stable, mice were trained individually to associate a tone with reward delivery. Finally, after training, mice competed for rewards during "reward competition" with cagemates of known social ranks. b. Mice learned the tone reward association at the same rate across social ranks. Latency to reward consumption following tone onset decreased over sessions, while the percent of trials with a latency of less than 10 seconds increased over sessions. Data are plotted as a function of social rank as measured by the tube test. c. Mice with higher relative ranks (dominant; dom) collected more rewards than subordinate (sub) when competing for the rewards ( $n=12$ pairs; paired t-test, $p=0.004$ ). Port occupation, pushing success, and time displaced from port was different across ranks ( $n=12$ dyads; paired t-test, occupation $p=0.04$; pushing success $p=0.02$; displaced $p=0.02$ ). $d$. Architecture of AlphaTracker which combines object detection using You Only Look Once (YOLO) bounding boxes, pose estimation using 
single animal pose estimator (SAPE), and identity-tracking based on intersection over union (IOU) for subsequent frames with an additional Kalman filter to correct tracking errors. e. AlphaTracker precision for tracking two unmarked, near-identical mice is higher than human precision separated by body parts, averaged across 3 replicates for different training set fractions (left) or in total (mean Average Precision over all body parts) (right) when training with $>100$ frames. f. Diagram depicting features used for AlphaTracker's unsupervised clustering of the tracking datapoints. The features include head length, body length, body-head angle, displacement of the nose, distance between mice, angle between mice. $\mathrm{g}$. Dendrogram and UMAP plot for unsupervised clustering of AlphaTracker output for the behavioral clusters from two interacting mice. h. Example frames from clips belonging to a specific cluster (cluster ID indicated with the color outline).
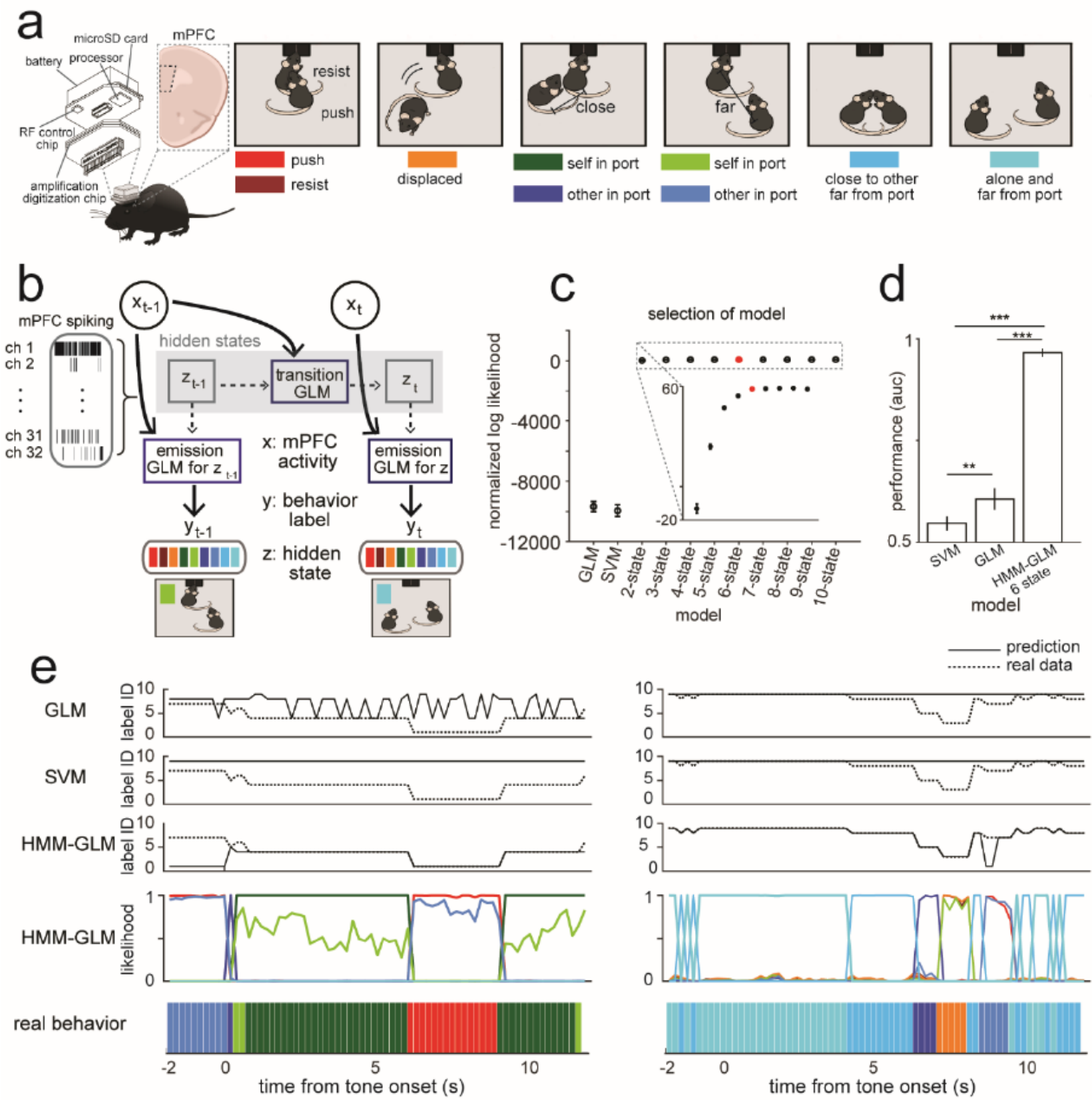

Figure 2 
A dynamic model with hidden states accurately predicts behavior from mPFC neural activity during social competition a. Left, wireless device used for MPFC extracellular recording. Right, social competition behavioral states used for the decoder models. Pushing, resisting, and displacing were validated by an experimenter while the rest of the behavioral states were determined based on AlphaTracker output. b. A Hidden Markov Model (HMM) was combined with generalized linear models (GLM) to model the relationship between neural activity and behavioral states. HMM transitions were determined with GLMs that used mPFC multi-unit spiking activity to predict the hidden state, and for each hidden state there was a unique emission GLM that used mPFC activity to predict the behavioral label. c. A model with 6 hidden states had the best performance (shown in red) based on normalized log likelihood for behavioral state predictions. Inset plot shows the data in the rectangle zoomed in which shows the HMM-GLM models and that improvement plateaus at 6 states. Error bars indicate standard error across 10 cross-validations. d. HMM-GLM decoded behavioral state from neural activity better than other classifiers (support vector machine and GLM) as measured using area under the receiving operator curve (AUC; $n=9$ behaviors; Wilcoxon rank-sum, $\left.{ }^{* *} p<.01, * \star * p<.001\right)$. Error bars indicate $95 \%$ confidence intervals across the decoding of the 9 behavioral states. e. Example trials with real behavioral states and predictions for the GLM, SVM and HMM-GLM 6-state model. 

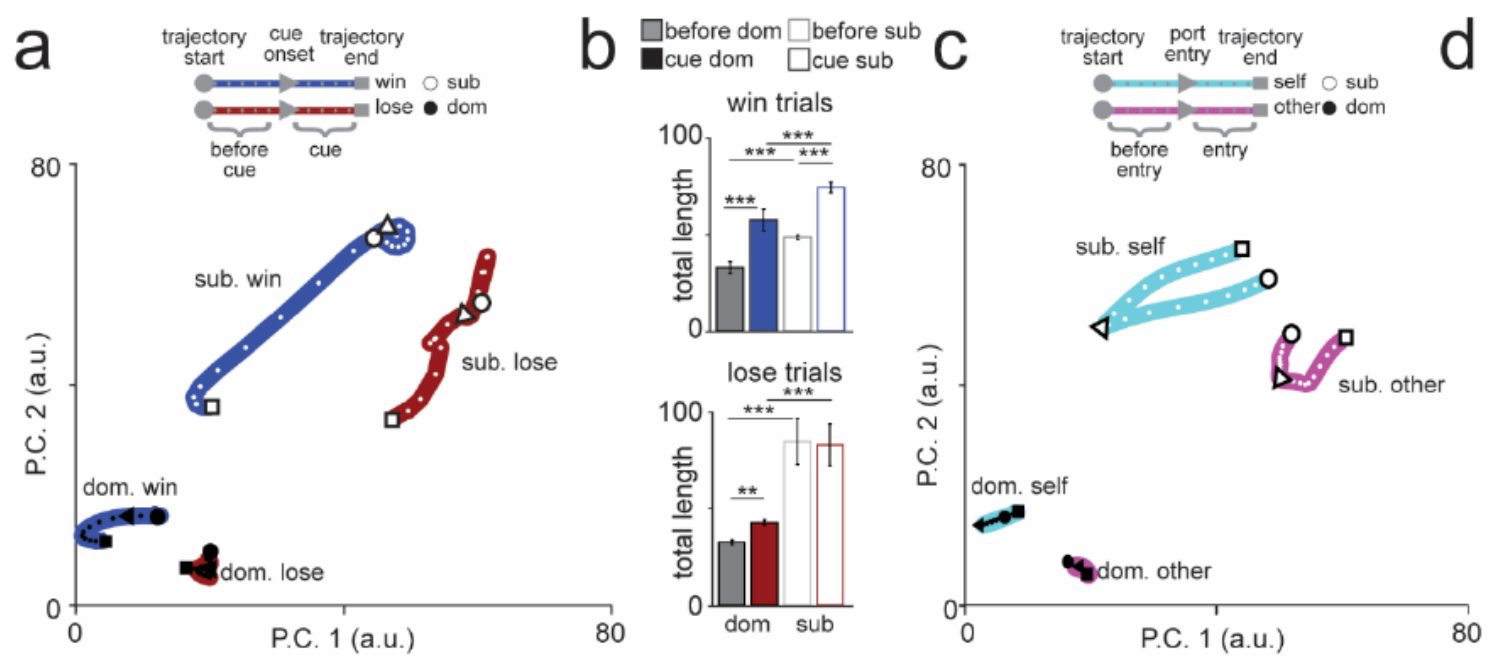

$\square$ before dom $\square$ before sub

entry dom $\square$ entry sub
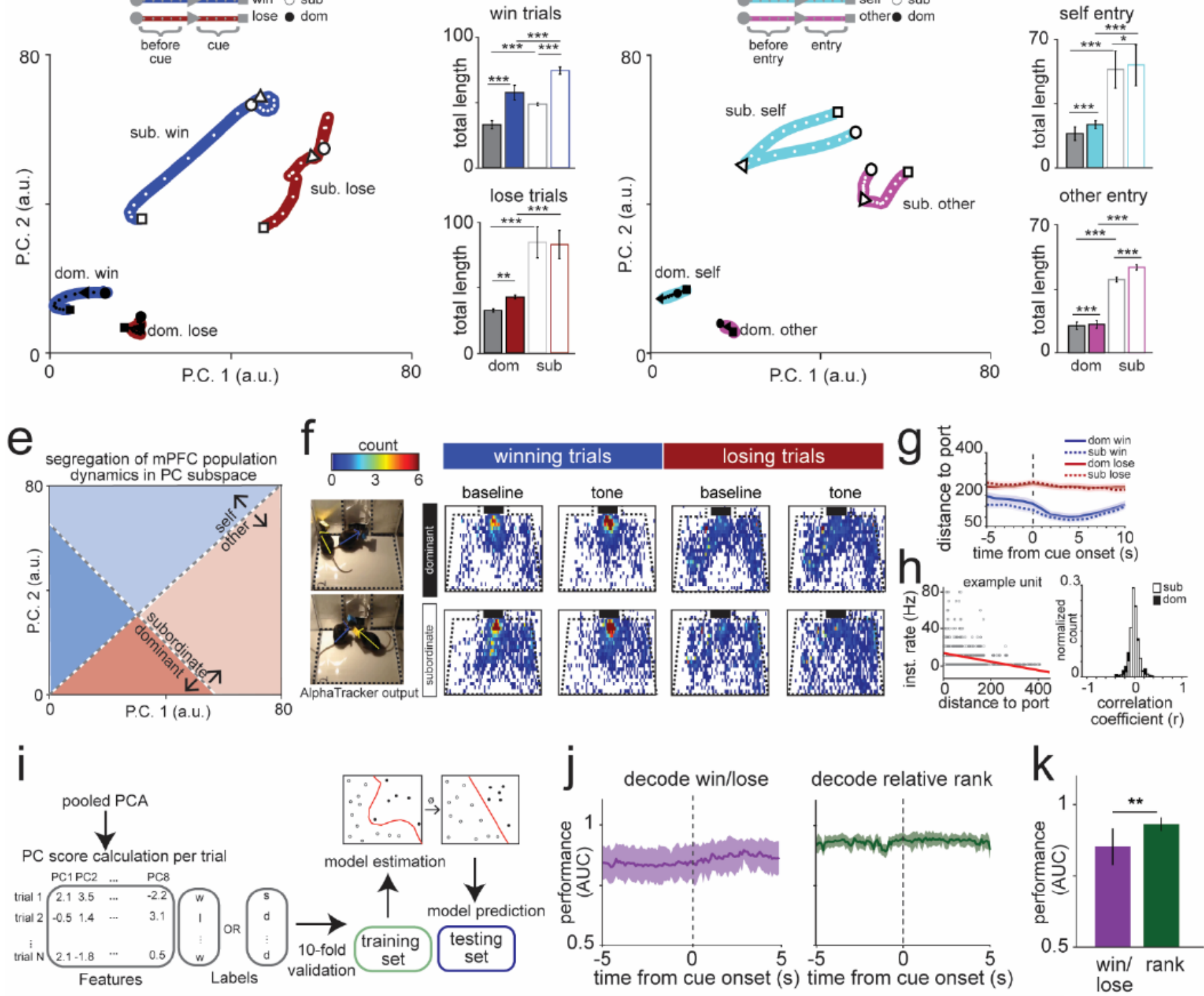

\section{Figure 3}

Social rank and competitive success is decoded by mPFC population dynamics a. Neural trajectories of mPFC population firing rate differ by rank and trial-type during tone presentation in a lower dimensional common principal component (PC) sub-space (neurons recorded from dominants: $n=507$ and subordinates: $n=490$ units). b. Trajectory lengths (using principal components that captured $90 \%$ of variance) for win (top) and lose trials (bottom) are highest for subordinates ( $n=13$ iterations; win 2-way ANOVA main effects of rank $F(1,50)=79.2, p=6 \times 9-12$ and event, $F(1,50)=193, p=8 \times 10-19$; lose 2-way ANOVA main effect of rank $F(1,50)=135, p=7 \times 10-16)$. $c$. Neural trajectories of mPFC population firing rate differ between dominant (dom) vs subordinate (sub) for port entries during the tone in a lower dimensional common PC subspace. d. Trajectory lengths (using PCs that captured $90 \%$ of variance) for self entry (top) and other entry (bottom) during the tone are higher for subordinates ( $n=13$ iterations; self entry 2-way ANOVA main effect of rank $F(1,50)=75, p=1 \times 10-11$; other entry 2-way ANOVA main effect of 
rank $F(1,50)=1,003, p=5 \times 10-35$, event $F(1,50)=18.1, p=0.00008$ and interaction $F(1,50)=11.2, p=0.0016)$. e. Schematic summarizing the neural trajectory segregation by rank and event type in the PC subspace. $f$. Average time spent in different parts of the chamber for win vs lose trials for the five seconds prior to tone vs first five seconds of tone. Black squares represent the reward port location. g. Distance to reward port differed by trial-type but not by rank (trials: dom win $=290$, dom lose $=349$, sub win $=349$, sub lose=290; 2-way ANOVA, main effect of trial-type $F(1,1274)=353, p=8.8 \times 10-70$, rank $p=0.098$ and interaction $\mathrm{p}=0.066)$. $\mathrm{h}$. Left, example unit with firing rate correlated to distance to reward port $(\mathrm{r}=-0.36$; $\mathrm{p}<.001$; red line indicates linear fit). Right, distribution of the correlation coefficients for firing rate and distance to port for the population of mPFC single units did not differ by rank (dom=321, sub=479; Kolmogorov-Smirnov test, $\mathrm{p}=0.48$ ). i. Support Vector Machine (SVM) data pipeline to decode rank or competition outcome based on single trial population mPFC data in the common behavioral subspace. j. SVM performance is higher than chance for decoding competitive success or rank (area under the receiving operating curve: AUC). Error bars indicate $95 \%$ confidence intervals from 10 -fold crossvalidations. $\mathrm{k}$. The decoder performs better when predict rank than competition outcome from single trial mPFC population activity ( $n=10$ fold validation; Wilcoxon rank-sum, $p=0.011)$. Error bars in panels a-d indicate $95 \%$ confidence intervals obtained by calculating the neural trajectories with the leave one out method leaving out data from one animal at a time. All post-hoc comparisons are Bonferroni-corrected ttests, ${ }^{\star \star} p<0.01,{ }^{\star \star \star} p<0.001$. All 2-way ANOVAs were for rank and event (baseline vs event) or rank and trial-type (win vs lose trial). 

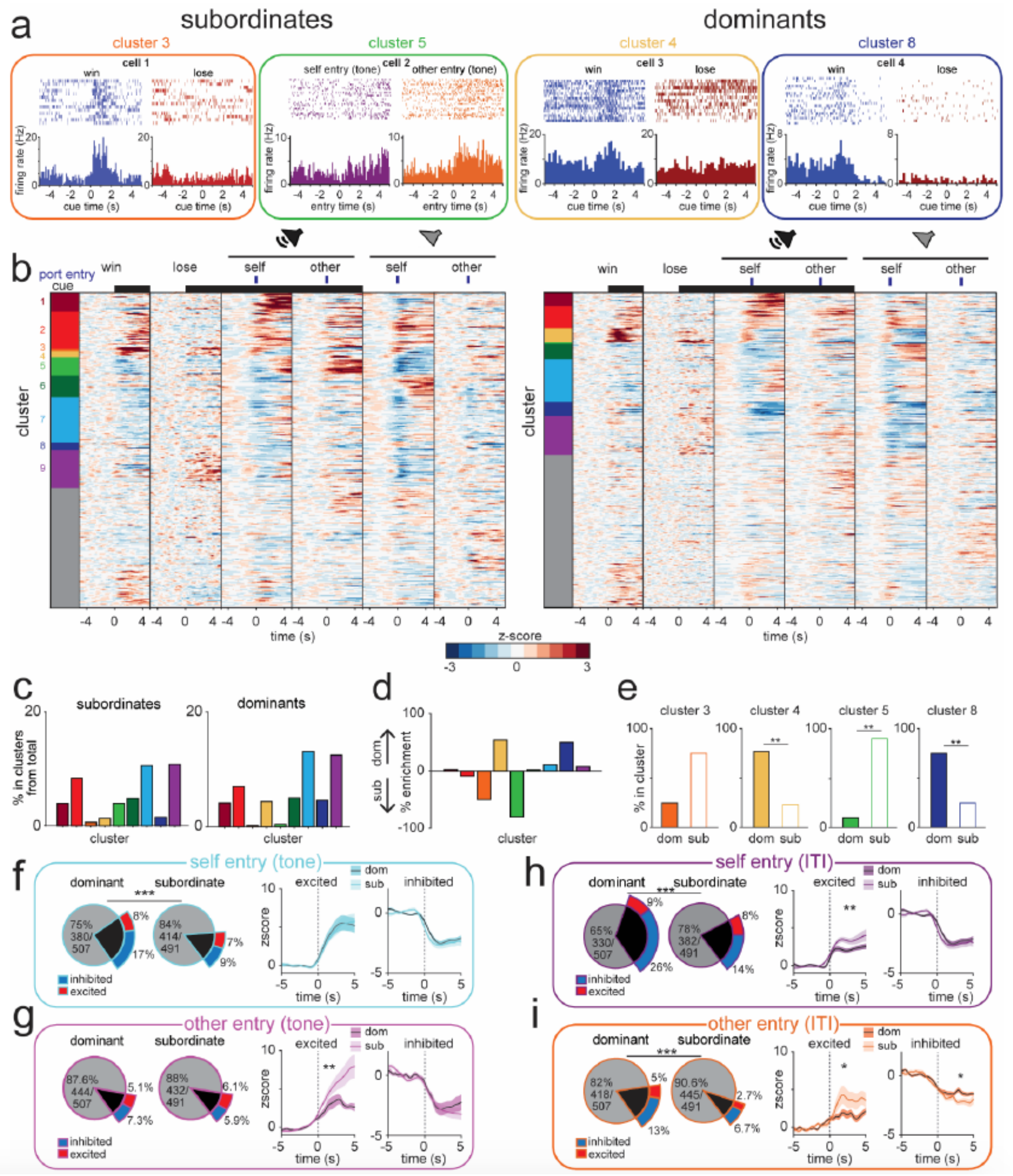

\section{Figure 4}

Individual mPFC cells show rank-dependent responses to task-relevant events during social competition a. Example cells from subordinate animals (left) responding to self and other port entries and from dominant animals (right) responding to win and lose trials differently. b. Heatmaps (left=subordinate; right=dominant) for firing rate responses to the six behavioral events. Color bar indicates functional clusters obtained by hierarchical agglomerative clustering. Clustering was performed with all cells, but 
only responsive cells are shown in the heatmap (mean firing rate was larger than 2 or smaller than -1 zscores to any behavioral event). c. Distribution of mPFC cells across functional clusters in subordinates (sub; left) and dominants (dom; right). d. Percent difference between dom and sub cells (\% enrichment) in functional cluster membership showed differences across ranks. e. Several clusters had more subordinate cells (cluster 3: Fisher's exact test, $p=0.48$; cluster 5: Fisher's exact test, $p=5 \times 10-7$ ) while others had more dominant cells (cluster 4: Fisher's exact test, $p=0.0002$; cluster 8: Fisher's exact test, $p=0.0004)$. $f$. Left, dominant mice had more responsive cells to self port entries during the tone (dom self $n=127 / 507$, sub self $n=63 / 491$; Fisher's exact test, $p=0.0002$ ). Right, response magnitude to self port entries during the tone had no rank difference (Wilcoxon rank-sum: excitation $p=0.28$ and inhibition $p=0.99)$. g. Left, there was no rank difference in the number of cells that respond to other port entries during the tone (dom other $n=63 / 507$, sub other $n=59 / 491$; Fisher's exact test, $p=0.84$ ). Right, response magnitude for subordinate excited cells was higher than dominant's response to other port entries (Wilcoxon rank-sum: excitation $\mathrm{p}=0.006$, inhibition $\mathrm{p}=0.11$ ). $\mathrm{h}$. Left, dominants had more responsive cells to self port entries during the inter-trial intervals (ITI) (dom self $n=177 / 507$, sub self $n=109 / 491$, Fisher's exact test, $p=9.8 \times 10-6)$. Right, subordinates had a larger excitation magnitude for self port entries during ITI (Wilcoxon rank-sum: excitation $p=0.006$ and inhibition $p=0.28$ ). i. Bottom left, dominants had more responsive cells to other port entries during ITI (dom other $n=89 / 507$, sub other $n=46 / 491$; Fisher's exact test, $p=0.00019)$. Right, subordinates had larger excitation and inhibition magnitude for other port entries during ITI (Wilcoxon rank-sum: excitation $\mathrm{p}=0.015$ and inhibition $\mathrm{p}=0.04$ ). 

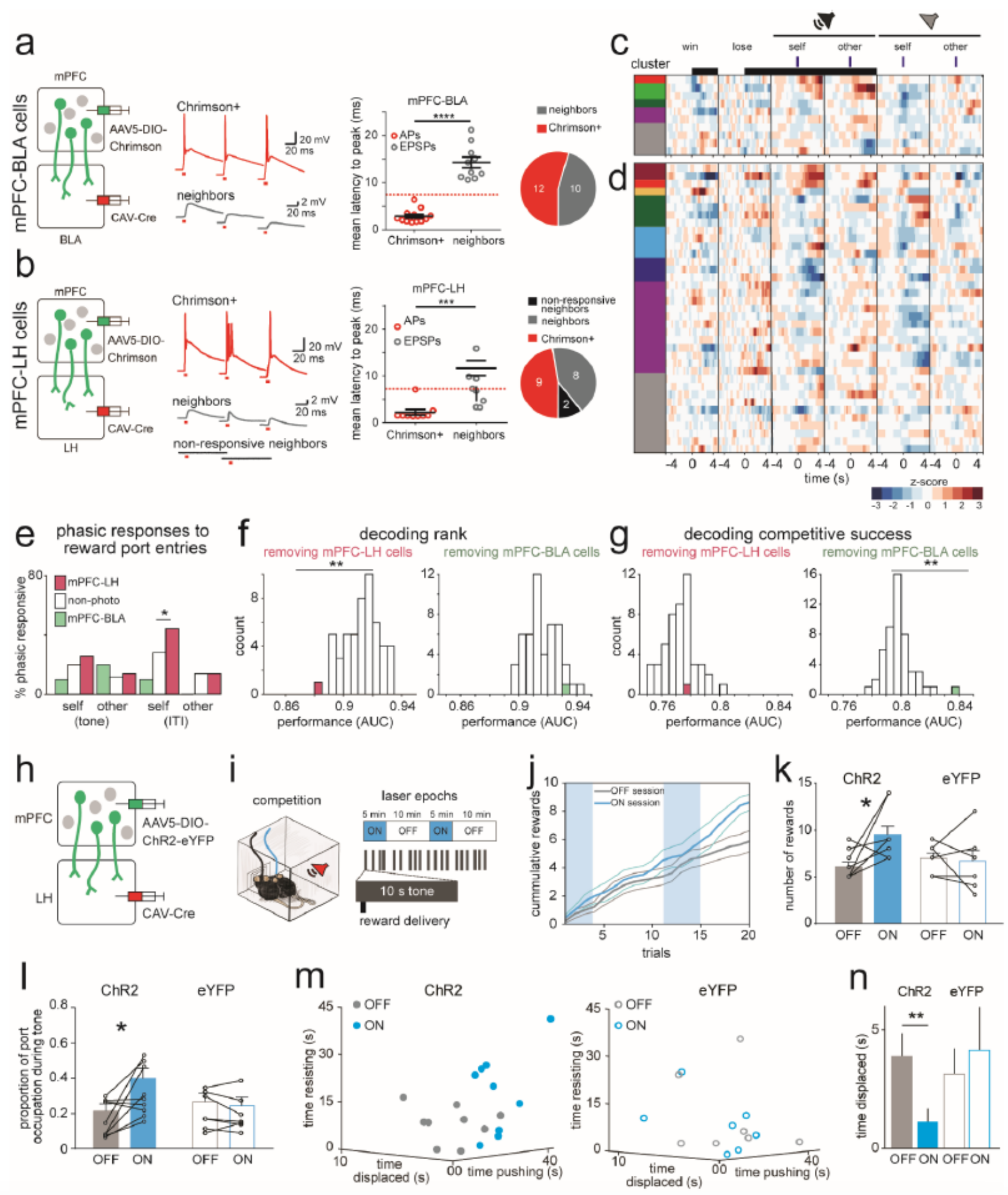

\section{Figure 5}

mPFC-LH pathway encodes social rank information and modulates social dominance behavior a. Viral strategy to stimulate mPFC-BLA cells using a dual viral approach. Left, example mPFC-BLA cell (Chrimson+) and neighbor cell (Chrimson-) responding to pulses of red light. Middle, average photolatency of action potentials (AP) or excitatory postsynaptic potentials (EPSPs) was lower for Chrimson+ cells compared to neighboring cells (Chrimson $+n=12$ vs neighbors $n=10$; t-test, ${ }^{\star \star \star \star} p<0.0001$ ). Dash line 
indicates latency threshold used for phototagging mPFC-BLA projectors. Right, number of Chrimson+ and neighbor cells that were responsive to light. b. Viral strategy to stimulate mPFC-LH cells. Left, example mPFC-LH cell (Chrimson+) and neighbor cell responding to pulses of red light. Middle, average photolatency was lower for Chrimson+ cells compared to neighboring cells (Chrimson+ $n=9$ vs neighbors $n=8$; $t-$ test, $* \star \star \star p<0.0001)$. Dash line indicates latency threshold used for phototagging mPFC-LH projectors. Right, number of Chrimson+ and neighbors that were responsive to light. c. Heat plot with firing rate responses to win, lose and port entries for MPFC-BLA $(n=10)$ and d. MPFC-LH $(n=43)$ phototagged cells during the reward competition assay. Color bars indicate functional cluster. e. mPFC-LH cells were more responsive to self entries during ITI than non-phototagged cells while mPFC-BLA cells did not differ from non-phototagged cells (self entries ITI: mPFC-LH $n=16 / 43$, non-phototagged $n=185 / 920$, Fisher's exact test, $p=0.011$; other entries ITI: mPFC-LH $n=12 / 43$, non-phototagged $n=150 / 920$, Fisher's exact test, $\mathrm{p}=0.058$ ). f. Removing mPFC-LH cells (left), but not mPFC-BLA cells (right), decreased social rank decoding performance as compared to removing a random subset of the same number of nonphototagged cells 50 times (plotted in white) (mPFC-LH Z-test, $p=0.004$; mPFC-BLA Z-test, $p=0.091$ ). g. Removing mPFC-LH cells did not affect win vs. lose decoding (left), while removing mPFC-BLA cells increased win vs. lose decoding performance (mPFC-LH Z-test, $p=0.68$; mPFC-BLA Z-test, $p<.0001$ ). $h$. Viral strategy to photo-stimulate mPFC-LH cells using Channelrhodopsin-2 (ChR2). i. During reward competition sessions consisting of light OFF or light ON sessions in which light was delivered in epochs (5 min light epoch consisting of four $5 \mathrm{~ms}$ light pulses at $100 \mathrm{~Hz}$ every $200 \mathrm{~ms}$ ). j. Cumulative rewards obtained by stimulated mice in the light OFF session vs light ON session. $\mathrm{k}$. MPFC-LH cell stimulation increased the number of trials won (chR2 $n=9$, eYFP $n=6$; 2-way RM ANOVA interaction of virus and light $F(1,14)=5.82, p=0.03$; Bonferroni corrected t-test ChR2 $p=0.01)$. I. mPFC-LH cell stimulation increased the time occupying the reward port during the tone (ChR2 $n=9$, eYFP $n=6$; 2-way RM ANOVA interaction of virus and light $F(1,14)=6.73, p=0.02$; Bonferroni corrected t-test, ChR2 $p=0.02)$. m. The combination of time spent pushing, resisting and being displaced was non-overlapping for light off and light on sessions for ChR2 mice, but overlaps in the eYFP group. $\mathrm{n}$. Time spent being displaced decreased with stimulation (ChR2 $n=9$ paired t-test, $p=0.005$; eYFP $n=6$ paired t-test, $p=0.28$ ).

\section{Supplementary Files}

This is a list of supplementary files associated with this preprint. Click to download.

- Methodsformanuscript20201015v2.docx

- SuppMovie1alphatracker.mp4

- SuppMovie2decoder.mp4

- SuppMovie3Taskevents.mp4

- ExtendedDataFiguresandlegends20201016.pdf 